\title{
Le « retour » du tramway en France, origines et controverses
}

The come back of Tramway in France, origins and controversies

\section{Sébastien Gardon et Harold Mazoyer}

\section{OpenEdition}

\section{Journals}

Édition électronique

URL : https://journals.openedition.org/rhcf/2129

DOI : 10.4000/rhcf.2129

\section{Éditeur}

Rails \& histoire

\section{Édition imprimée}

Date de publication : 2 avril 2014

Pagination : 105-114

ISSN : 0996-9403

Référence électronique

Sébastien Gardon et Harold Mazoyer, «Le « retour » du tramway en France, origines et controverses », Revue d'histoire des chemins de fer [En ligne], 45 | 2014, mis en ligne le 29 mai 2015, consulté le 22 avril 2022. URL : http://journals.openedition.org/rhcf/2129; DOI : https://doi.org/10.4000/rhcf.2129 
Sébastien GARDON et Harold MAZOYER

\section{Le " retour " du tramway en France, origines et controverses}

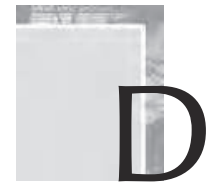

epuis le printemps 2013, un nouveau séminaire intitulé «Réinventer le tramway ? Quarante années de TCSP, tramways et transports guidés en France : controverses et réalisations " est organisé par l'Association pour l'histoire des chemins de fer (AHICF) et l'UMR AUSser (CNRS et École nationale supérieure d'architecture de Paris-Belleville). L'objectif de ce séminaire est de mettre en lumière les processus qui ont participé au " retour » du tramway dans les grandes villes françaises et à l'exportation du «tramway français standard » dans nombre d'autres métropoles. Les échanges, déjà engagés au cours de six séances, à la fois riches et intenses, permettent de mettre en question les enjeux qui nourrissent les controverses nées aux échelons locaux, nationaux et internationaux. Ainsi les présentations et discussions reviennent sur les acteurs qui ont favorisé le retour en grâce du tramway, sur l'articulation dans les processus de décision entre les temps des projets et le calendrier électoral, sur les ressorts des options technologiques choisies, sur le lien entre politiques d'aménagement urbain et projets de transport ou, enfin, sur les logiques professionnelles qui sous-tendent la mise en place et l'exploitation de ces nouvelles lignes de tramway. 
En France, on recense aujourd'hui vingt-six réseaux de tramways ${ }^{1}$ (tableau 1 et carte 1). Pourtant, jusqu'au début des années 1970, le développement des transports en ville s'organise autour de l'automobile et des voiries routières et autoroutières ${ }^{2}$. Après le démantèlement des derniers réseaux de tramways hérités du xix ${ }^{\mathrm{e}}$ siècle dans les années $1950^{3}$ (hormis à Lille, Marseille et Saint-Étienne), un tournant se situe donc au cours de la décennie 1970 qui est souvent considérée comme l'origine d'un regain d'intérêt pour les transports collectifs ${ }^{4}$. En effet, au cours de cette période, Paris aménage son RER (Réseau Express Régional), Lyon et Marseille construisent leur réseau de métro, Lille prépare la mise en place de son VAL ("véhicule automatique léger ", métro automatique) et les projets de tramways sont à nouveau étudiés. Le contexte, marqué par la crise économique et énergétique, est favorable à la recherche de nouvelles solutions tant technologiques qu'institutionnelles ou économiques Les mobilisations locales, sociales ou environnementales, et le foisonnement des réflexions sur les transports urbains jouent également un grand rôle dans cette dynamique : création du Groupement pour l'étude des transports urbains modernes (GETUM) en 1967, création de l'Institut de recherche sur les transports (IRT) en 1970, colloque de Tours des 25 et 26 mai 1970 qui débouche sur un manifeste pour les transports urbains 6 , rapport du Club de Rome en 1972, étude « Neuchateau » de la SOFRETU RATP en 1973-19757.

À l'origine de ce renouveau du tramway, on trouve également une initiative du secrétaire d'État aux Transports (gouvernement de Jacques Chirac), Marcel Cavaillé. Le 27 février 1975, il adresse une lettre aux maires de huit villes : Bordeaux, Grenoble, Nancy, Nice, Rouen, Strasbourg, Toulon et Toulouse, par laquelle il les incite à réfléchir à des choix techniques mobilisant des matériels " comparables à des tramways modernes". Ce que l'on appelle

1- Bientôt 28 avec les réseaux de Besançon et Aubagne ouverts en 2014. Trois autres projets sont également prévus à Amiens, Avignon, Grand Genève. Les projets de Lens et de Toulon ont été abandonnés. Des projets sont en discussion à Dunkerque et à Nîmes.

2- Voir Jean Poulit, «La circulation urbaine », Revue générale des routes et des aérodromes, n 462 (1971), p. 59-78.

3- Voir Pierre-Henri Emangard, "Les tramways en Europe, Une vision diachronique ", Transports urbains, $\mathrm{n}^{\circ} 120$ (juin 2012), p. 3-8.

4- Voir sur ce point les travaux de Jean-Marc OfFner ou de Christian Lefèvre et notamment Les Transports urbains en question, Usages-Décisions-Territoires, Paris, Celse, 1990.

5- La mise en place progressive du « versement transport » pour les villes de province à partir de 1973 donne de nouveaux moyens aux villes pour développer leurs réseaux de transports en commun. 6- Livre Vert: Les transports collectifs urbains. Colloque de Tours, 25-26 mai 1970, numéro spécial d'Équipement, logement, transports, n ${ }^{\circ}$ 48-49 (mars-avril 1970).

7- Voir Christian GÉrondeAu, Les Transports urbains, Paris, PUF, Collection « Que-sais-je ?», 1977. 
Tableau 1. Les réseaux de tramways dans les villes françaises*.

\begin{tabular}{|c|c|c|c|c|}
\hline Ville & Date d'inauguration & \begin{tabular}{|c} 
Taille du \\
réseau en $\mathrm{km}$
\end{tabular} & Nombre de lignes & Caractéristiques \\
\hline Saint-Étienne & $\begin{array}{c}1881 \text { (ancien réseau) / } \\
1983\end{array}$ & 11,7 & 3 lignes & \\
\hline Marseille & $\begin{array}{c}1893 \text { (ancien réseau) / } \\
2007\end{array}$ & 12,6 & 2 lignes et une $3^{e}$ en projet & Aérien et souterrain \\
\hline Lille & $\begin{array}{c}1909 \text { (ancien réseau) / } \\
1994\end{array}$ & 22 & 2 lignes & Aérien et souterrain \\
\hline Nantes & 1985 & 41,3 & $\begin{array}{c}3 \text { lignes (projets d'extension et } \\
\text { de nouvelles lignes) }\end{array}$ & \\
\hline Grenoble & 1987 & 29,6 & $\begin{array}{c}4 \text { lignes (projets d'extension et } \\
\text { une } 5^{\mathrm{e}} \text { en construction) }\end{array}$ & \\
\hline île-de-France & 1992 & 71,6 & $\begin{array}{c}6 \text { lignes (plusieurs lignes en } \\
\text { projet) }\end{array}$ & \\
\hline Strasbourg & 1994 & 38,7 & 6 lignes (projets d'extensions) & Extension vers l'Allemagne \\
\hline Rouen & 1994 & 15,2 & 2 lignes & Aérien et souterrain \\
\hline Montpellier & 2000 & 56 & $\begin{array}{c}4 \text { lignes (projets d'extension de } 2 \\
\text { autres lignes) }\end{array}$ & \\
\hline Nancy & 2000 & 11,1 & 1 ligne & Pneumatique \\
\hline Orléans & 2000 & 29,3 & 2 lignes & $\begin{array}{l}\text { Portion avec système } \\
\text { d'alimentation par le sol }\end{array}$ \\
\hline Lyon & 2001 & 61,8 & 6 lignes (projets d'extension) & $\begin{array}{l}\text { Une ligne directe vers } \\
\text { l'aéroport }\end{array}$ \\
\hline Caen & 2002 & 15,7 & $\begin{array}{c}2 \text { lignes (2 autres lignes en } \\
\text { projet) }\end{array}$ & Pneumatique \\
\hline Bordeaux & 2003 & 44 & $\begin{array}{c}3 \text { lignes (projets d'extension et } \\
\text { d'une } 4^{\mathrm{e}} \text { ligne) }\end{array}$ & $\begin{array}{c}\text { Portion avec système } \\
\text { d'alimentation par le sol }\end{array}$ \\
\hline \begin{tabular}{|l|} 
Clermont- \\
Ferrand \\
\end{tabular} & 2006 & 14,2 & $\begin{array}{c}1 \text { ligne (projet d'extension et } \\
\text { d'une } 2^{\mathrm{e}} \text { ligne) }\end{array}$ & Pneumatique \\
\hline Mulhouse & 2006 & 16,2 & 3 lignes (projets d'extension) & \\
\hline Valenciennes & 2006 & 18,3 & $\begin{array}{c}1 \text { ligne (une } 2^{\mathrm{e}} \text { ligne en } \\
\text { construction) }\end{array}$ & \\
\hline Le Mans & 2007 & 15,4 & 1 ligne (une $2^{\mathrm{e}}$ ligne en projet) & \\
\hline Nice & 2007 & 9,2 & $\begin{array}{c}1 \text { ligne (projets d'extension et de } \\
2^{\mathrm{e}} \text { ligne) }\end{array}$ & \\
\hline Toulouse & 2010 & 10,9 & $\begin{array}{c}1 \text { ligne (plusieurs lignes en } \\
\text { projet) }\end{array}$ & \\
\hline Reims & 2011 & 11,2 & 2 lignes & \\
\hline Angers & 2011 & 12,3 & 1 ligne (projet d'une $2^{\mathrm{e}}$ ligne) & $\begin{array}{l}\text { Portion avec système } \\
\text { d'alimentation au sol }\end{array}$ \\
\hline Brest & 2012 & 14,3 & $\begin{array}{c}1 \text { ligne (projet d'extension et } \\
\text { d'une } 2^{\mathrm{e}} \text { ligne) }\end{array}$ & \\
\hline Dijon & 2012 & 19 & 2 lignes & \\
\hline Le Havre & 2012 & 13 & 2 lignes & \\
\hline Tours & 2013 & 15,5 & 1 ligne (une $2^{\mathrm{e}}$ ligne en projet) & $\begin{array}{l}\text { Portion avec système } \\
\text { d'alimentation au sol }\end{array}$ \\
\hline
\end{tabular}

* Il faut également noter qu'une ligne du réseau de tramway de Bâle (Suisse) pénètre jusqu’à la commune française de Leymen (Haut-Rhin). 
Figure 1. Les réseaux de TCSP en service ou en projet dans les villes françaises en 2013.

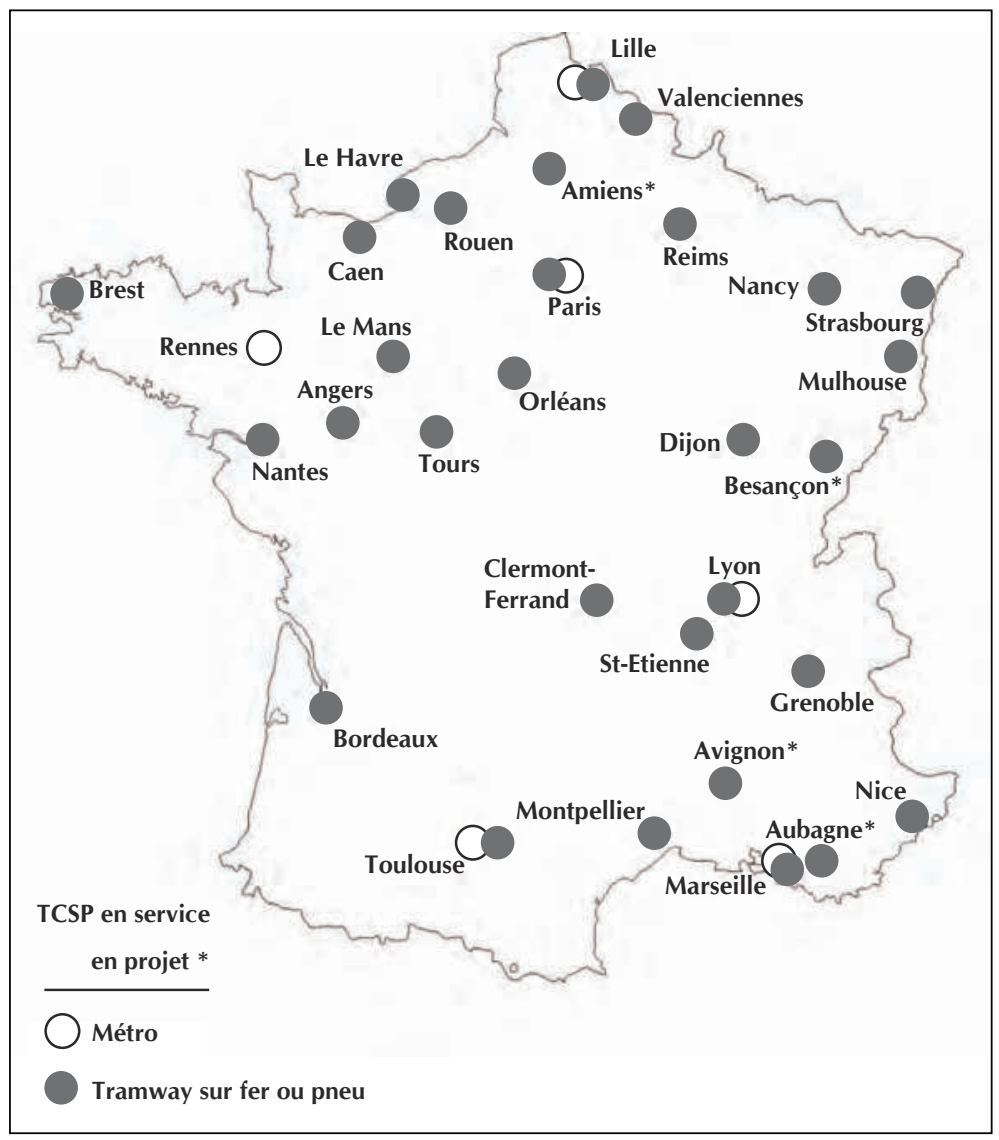

désormais le "Concours Cavaillé " ${ }^{8}$ prend la forme d'un cahier des charges diffusé le 25 août 1975 aux industriels du secteur des transports en commun dont les lauréats sont désignés le 2 avril $1976^{9}$. Il transforme durablement les projets de transports et d'aménagement des villes françaises au cours des décennies suivantes. Il est souvent considéré comme l'acte de naissance de ce

8- Deux acteurs majeurs de cette période avaient été contactés pour participer au séminaire mais n'ont pas pu hélas se joindre à nos échanges : Marcel Cavaillé lui-même et Raymond Guitard (chef de cabinet de Marcel Cavaillé), tous deux décédés en 2013.

9- Sur cet épisode du concours Cavaillé, nous renvoyons à l'article de Benoît Demongeot : « "Adapter le transport collectif à la ville telle qu'elle est." Les initiatives Cavaillé de 1975 ou le retour du tramway sur l'agenda national » dans ce même numéro. 
que l'on qualifie rétrospectivement de " tramway français standard ». Mais c'est finalement la ville de Nantes ${ }^{10}$, non sollicitée au départ, qui est la première à participer au renouveau du tramway dans les villes françaises avec la mise en service de sa première ligne en $1985^{11}$.

Ainsi la solution "tramway " (re)devient progressivement crédible aux yeux des élus, des experts, des professionnels du transport. Pourtant, elle n'est pas encore envisagée comme le moyen de lutter activement contre la congestion des villes par l'automobile. Le tramway se développe là où existent des possibilités, notamment des réserves foncières, comme à Nantes. Il est d'abord aménagé à côté de la circulation automobile. Au-delà d'un modèle français, alors en construction, les orientations prises successivement par les villes françaises qui se tournent à nouveau vers le tramway offrent des aspects parfois différents sur le plan local, en fonction des contextes d'émergence des projets $^{12}$. Ainsi sont débattues systématiquement, avec des réponses parfois différentes, les questions des alternatives techniques (tracé au sol, en viaduc ou souterrain, matériel sur rail, sur pneus, système d'alimentation électrique, etc. ${ }^{13}$ et les grandes options d'aménagement (desserte du centre ou du périurbain, parcs-relais). Entre le milieu des années 1970 et le milieu des années 1980, les controverses sont encore fortement marquées par le désir de mettre en œuvre des solutions technologiques radicalement nouvelles et le "vieux " tramway doit (dé)montrer sa " modernité ». Les débats prennent nécessairement une tournure politique à travers le positionnement des élus vis-à-vis des projets, les enjeux électoraux qu'ils représentent et les mobilisations locales qu'ils suscitent. En ce sens, un premier "virage " symbolique est franchi à partir du projet de Strasbourg longuement débattu aux cours des élections de 1989 et qui montre aux élus que l'on peut gagner une élection avec le tramway ${ }^{14}$. Le rythme des projets qui suivent dépend fortement du calendrier électoral : débats sur le projet pendant la campagne électorale, lancement des études en début de mandat, travaux en cours de mandat et inauguration souhaitée avant les prochaines élections, etc. La question des aménagements et des

10- Voir Michel Bigey, Les Élus du tramway, Mémoire d’un technocrate, Paris, Éditions Lieu commun, 1993. 11- En réalité c'est l'extension du tramway au sud de la ville de Saint-Étienne en 1983 qui marque les premiers aménagements en faveur du tramway en France depuis la Deuxième Guerre mondiale. 12- Voir le numéro 98 de la revue Transports urbains, «Le tramway et la ville», janvier-mars 1998. 13- Robert Marconis, « Métros, VAL, Tramways... La réorganisation des transports collectifs dans les grandes agglomérations de province en France », Annales de géographie, vol. 106 (1997), $\mathrm{n}^{\circ}$ 593-594 (janvier-avril 1997), p. 129-154.

14- Voir sur ce point les interventions de Benoît Demongeot, Frédéric Héran et Philippe Hamman lors des trois premières séances du séminaire (enregistrements en ligne sur le site www.ahicf.com).. 
projets urbains (requalification des espaces, insertion paysagère, standardisation des projets, émergence de projets qui dépassent la solution de transport adoptée) prend de plus de place, notamment à partir des années 1990. Des métiers nombreux et d'une grande variété se trouvent donc impliqués dans la conduite des projets : concepteurs, ingénieurs, urbanistes, architectes, paysagistes, industriels, membres des administrations, gestionnaires, conducteurs, etc. Au-delà des rôles répartis entre maîtres d'œuvre et maîtres d'ouvrage, les services techniques des villes et les agences d'urbanisme participent activement à cette dynamique. Au fur et à mesure de leur développement, les projets de tramway tiennent compte davantage des enjeux intercommunaux et des relations entre le centre et les quartiers ou communes périphériques ${ }^{15}$. D'autres questions viennent se greffer sur cette problématique qui peut également prendre une tournure sociale, à travers les publics visés, l'insertion dans les quartiers, la tarification ou à partir de la capacité du tramway à réduire les inégalités urbaines. Les enjeux économiques et fonciers des projets sont également perceptibles, notamment à travers le rôle que peuvent jouer dans les décisions commerçants ou promoteurs immobiliers.

Au-delà des dynamiques locales, un pilotage national, voire européen, des projets transparaît à travers, notamment, la recherche de financements. Se pose alors la question des échelles de gouvernement et des relations entre les niveaux institutionnels impliqués au niveau local, national, européen. À travers ses appels à projets et les financements correspondants qu'il apporte (en moyenne 17 à $18 \%$ du coût des projets sélectionnés) l'État a en effet contribué, via l'implantation du tramway dans certaines villes, au développement d'une véritable filière économique et industrielle autour de plusieurs opérateurs. Cette filière qui s'appuie sur le " tramway français standard " élaboré par Alstom est de plus en plus exportatrice vers l'étranger (Europe, Afrique du Nord, MoyenOrient) et permet à plusieurs de ces projets de revendiquer une certaine French Touch. À la différence des réseaux français où aménagements et matériels roulants sont élaborés par des acteurs différents, à l'étranger ce sont plutôt des projets " clés en main " qui se négocient (où le matériel roulant ne compte que pour $15 \%$ de la facture totale) ${ }^{16}$. Mais ces différents succès masquent aussi d'autres choix et des échecs survenus dans des villes où le tramway n'a pas pu encore s'implanter (comme à Dunkerque, Lens, Nîmes ou Toulon) ou a connu quelques difficultés et retards (comme à Reims ou Nancy). Si la plupart des grandes villes

15- Voir sur ce point les interventions de Caroline Galdez, Cyprien Richer et Marc Wiel lors de la séance 4 du séminaire, le 11 décembre 2013.

16- Voir sur ce point l'intervention d'Emmanuel Bois (Alstom) lors de la séance 1 du séminaire, le 3 avril 2013. 
françaises sont aujourd'hui dotées d'un réseau de tramway, le développement de celui-ci passe à présent par les villes moyennes, comme en témoigne l'ouverture en 2014 d'un tramway à Aubagne (nouvelle génération Citadis avec des rames de 22 mètres), ou par l'essor encore timide en France des projets de tram-train ${ }^{17}$.

\section{Présentation du séminaire}

Le séminaire est animé par Sébastien Gardon et Harold Mazoyer, docteurs en science politique, et préparé par un groupe de travail qui en assume la responsabilité scientifique, composé de :

- Francis Beaucire, professeur émérite, université Paris 1 - Panthéon-Sorbonne

- Karen Bowie, professeure, histoire et cultures architecturales, ENSA Paris - La Villette, directrice AHTTEP (UMR AUSser 3329)

- Xavier Desjardins, maître de conférences, université Paris 1 - Panthéon-Sorbonne

- Pierre-Henri Emangard, maître de conférences associé à l'université du Havre, Responsable Pôle transports et déplacements, CNFPT La Rochelle

- Anne Grillet-Aubert, maitre-assistante, chercheuse à l'IPRAUS (UMR AUSser 3329), ENSA Paris-Belleville

- Michèle Merger, chargée de recherche honoraire au CNRS

- Arnaud Passalacqua, maître de conférences, université Paris-Diderot

- Pierre Zembri, professeur, université Paris Est, directeur du Laboratoire Ville Mobilité Transport

\section{Cycle 1 : La mise en place du tramway français : origines et controverses}

Séance 1 ( 3 avril 2013) : Le concours Cavaillé et la naissance du "Tramway français standard ». Les premiers projets de Nantes et Grenoble

- Arnaud Passalacqua, maître de conférences, université Paris Diderot, président de séance

- Benoît Demongeot, docteur en science politique

- Michel Bigey, auteur de Les Élus et le tramway, mémoires d'un technocrate

- Pierre-Henri Emangard, maitre de conférences associé à l'université du Havre

- Emmanuel Bois, Alstom Transport

Séance 2 (12 juin 2013) : Surface ou souterrain. Les cas de Strasbourg et de Toulouse

- Francis Beaucire, professeur émérite à l'université Paris 1, président de séance

- Jean Frébault, ingénieur général des Ponts et Chaussées

- Frédéric Héran, maître de conférences à l'université Lille 1

- Robert Marconis, professeur émérite à l'université Toulouse 2

17- Voir sur ce point Transports urbains, « Tram-Train et territoires », n 119 (octobre 2011). 


\section{Cycle 2 : Le tramway comme projet urbain}

Séance 3 (23 octobre 2013) : Du projet de transport au projet urbain : les liens entre urbanisme et transport

- Anne Aubert-Grillet, maître-assistante à l'ENSA Belleville, présidente de séance - Alfred Peter, directeur de l'atelier Alfred Peter

- Nadia Arab, maître de conférences à l'IUF

- Philippe Hamman, professeur de sociologie à l'université de Strasbourg

Séance 4 (11 décembre 2013) : L'intercommunalité, un accélérateur pour le tramway? Le compromis politique et territorial autour des transports

- Xavier Desjardins, maître de conférences à l'université Paris 1, président de séance

- Marc Wiel, urbaniste, ancien directeur de l'agence d'urbanisme de Brest, auteur de La Transition urbaine ou le passage de la ville pédestre à la ville motorisée

- Caroline Gallez, chercheuse à l'IFSTTAR

- Cyprien Richer, chercheur au CETE Nord-Picardie

Séance 5 (13 février 2014) : Journée d'étude sur le tramway en Seine-Saint-Denis

Séance commune avec le séminaire "La Région parisienne, territoires et cultures ", proposé par la RATP, le Centre d'histoire sociale de l'université Paris 1 et les Archives départementales de la Seine-Saint-Denis, reçue par la Maison de la RATP.

Matinée : Séminaire "La Région parisienne, territoires et cultures "

Les tramways et leur impact urbain : I'exemple du T1

- "L'histoire du tramway en Ile-de-France ", Jean-Emmanuel Terrier, historien des transports, responsable du développement des publics, Musée de l'Air et de l'Espace - "Le Conseil général de la Seine-Saint-Denis et le projet de T1 », Jean-Marie Deyherassary, chef du Service Aménagement-Transports au Département de la Seine-Saint-Denis

- "Le Tramway de Saint-Denis à Bobigny, un condensateur urbain ", Gilles Margot-Duclos, architecte associé de l'agence MDAA, précédemment chef du projet tramway au sein de l'agence Chemetov

Après-midi : Séminaire "Réinventer le tramway ? Quarante années de TCSP, tramways et transports guidés en France : controverses et réalisations "

Projet politique et enjeux sociaux : Le tramway en Seine Saint-Denis

- Aurélien Delpirou, maître de conférence à l'Institut d'Urbanisme de Paris et chercheur au sein du Lab'Urba (université Paris Est), président de séance

- Philippe Ventéjol, RATP, département Développement, Innovation et Territoires

- Elisabeth Gavrilovic, directrice de projet, PRU Clichy Montfermeil 
- Erwan Lamouroux, responsable Prospectivel Mobilité / Transport à la Communauté d'agglomération de Clichy-sous-Bois/Montfermeil

- Alain Schumacher, vice-président chargé des transports à la Communauté d'agglomération de Clichy-sous-Bois/Montfermeil.

Séance 6 (9 avril 2014) : Le tramway à Paris

- «Formes et figures des premiers tramways parisiens (1855-1938) : analyse sociale, urbaine et technique d'un système de transport déchu. ", Arnaud Passalacqua, Laboratoire Identités, Cultures, Territoires, université Paris Diderot - "Le tramway des Maréchaux ", Philippe Ventéjol, RATP, département Développement, Innovation et Territoires

- Pierre Zembri, Laboratoire Ville Mobilité Transport - Institut français d'urbanisme, président de séance

- « La fabrique politique du tramway parisien où les contorsions d'une solution en quête de problème, d'alliés et d'un "décideur" ", Philippe Zittoun, Laboratoire d'Économie des transports - École nationale des travaux publics de l'État

Programme (provisoire) des semestres suivants :

\section{Cycle 3 : La mobilité à l'issue de quarante ans de tramways}

Séance 7 : La question des métiers et la formation d'une culture professionnelle : le travail entre architectes, ingénieurs et paysagistes et les relations avec les services techniques des villes.

Séance 8 : Les effets territoriaux du tramway : valeur foncière et enjeux commerciaux. Les effets d'homogénéisation et de diversité du tramway.

Séance 9 : Rail ou pneu : retour sur les questions de terminologie.

Séance 10 : Les conducteurs : enjeu de statuts et conflits sociaux.

Séance 11 : Temps politique et temps des projets.

Séance 12 : Construction et circulation du modèle français de tramway.

Le séminaire compte deux séances par semestre, de 2013 à 2015. Il s'adresse aux chercheurs en architecture, urbanisme et transports et plus largement en sciences sociales comme aux professionnels de l'aménagement et du transport public, auxquels il propose de mieux connaître la suite des décisions locales et nationales qui ont conduit à des choix techniques, d'organisation et de services.

Les séances réunissent les points de vue de grands témoins (maitres d'ouvrage et maîtres d'œuvre, élus, techniciens, concepteurs, industriels, experts) et des points de vue de chercheurs spécialistes des transports urbains. 
L'enregistrement des interventions est disponible en ligne sur le site www.ahicf.com

Les entretiens approfondis menés avec les témoins seront mis en ligne sur la plate-forme Mémoire de l'industrie et des réseaux

www.memoire-orale.org

Les séances ont lieu les mercredis de 14 h à 17 h à l'École nationale supérieure d'architecture de Paris-Belleville, 60, boulevard de la Villette, 75019 Paris $\left(\mathrm{M}^{\circ}\right.$ Belleville), sauf mention contraire.

> Contact et inscriptions : seminaire_tramway@ahicf.com 